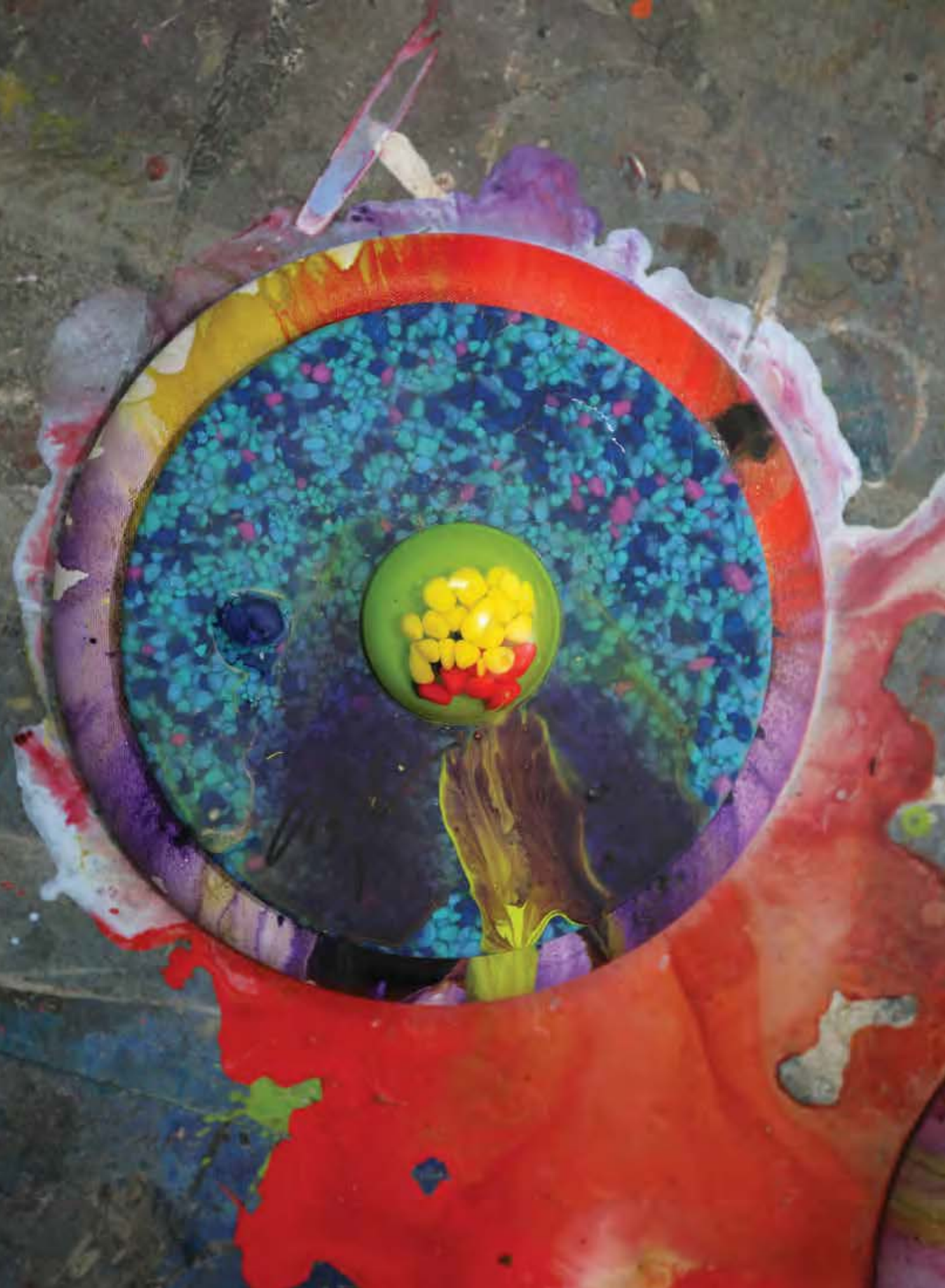


La Prímavera Besaba

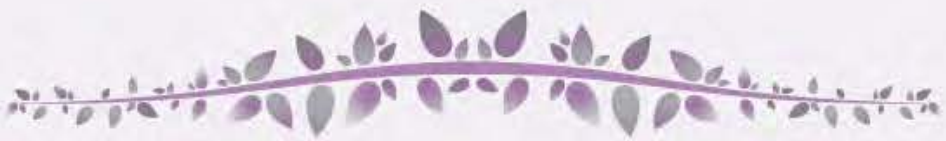

La primavera besaba suavemente fa arboleda, y el verde nuevo brotaba como una verde humareda.

Las nubes iban pasando sobre el campo juvenil... Yo vi en las hojas temblando las frescas Iluvias de abril.

Bajo ese almendro florido, todo cargado de flor -recordé-, yo he maldecido mi juventud sin amor.

Hoy en mitad de la vida, me he parado a meditar... Juventud nunca vivida, quién te volviera a soñar!

Antonio Machado

Poeta Español 


\title{
Jóvenes Universitarios ante la Formación Profesional y la Precariedad Laboral ${ }^{1}$
}

\author{
BLANCA NOEMÍ SILVA GUTIÉRREZ \\ AMADA LYDIA RODRÎGUEZ TÉLLEZ \\ Recibido 10.05.16 / Aprobado 11.08.16
}

RESUMEN. En esta investigación se estudió la situación laboral de la población estudiantil del Centro Universitario de Ciencias Económico Administrativas (CUCEA) de la Universidad de Guadalajara, Jalisco, México. El objetivo consistió en analizar las características de los empleos remunerados en los que se desenvuelven los estudiantes del CUCEA, así como el perfil sociodemográfico de los participantes. Estos jóvenes atienden las actividades y exigencias institucionales propias de la vida académica y al mismo tiempo buscan mejorar su situación tanto económica como de formación profesional, por lo que se ven en la necesidad de estudiar y trabajar de manera simultánea. El estudio se realizó con metodología de carácter cuantitativo y corte transversal, se aplicó una encuesta a los estudiantes del nivel licenciatura del CUCEA, durante el calendario 2015 " $B$ ". Entre los resultados se identificó la existencia de precariedad laboral respecto a los siguientes aspectos: la percepción salarial, la jornada laboral, la falta de prestaciones sociales así como la falta de contrato de trabajo.

Palabras clave: jóvenes universitarios, precariedad laboral, formación profesional.

\begin{abstract}
This study aims to investigate the status of labor and employment among the student population of the University Center of Economic and Administrative Sciences (CUCEA) at the University of Guadalajara in Jalisco, Mexico. The objective consisted in analyzing the characteristics of remunerative positions occupied by CUCEA students as well as the respective sociodemographic profiles of participants. These students attend to the activities and institutional demands of their academic lives while working to improve their economic and professional circumstances. The study was conducted through quantitative methods and transversal cuts. A questionnaire was given to undergraduate students at CUCEA during calendar year 2015 "B." Among the results obtained was the prevalence of precariousness employment in regard to the following characteristics: projected salaries, working hours, a lack of welfare benefits, and a scarcity of labor contracts.
\end{abstract}

Keywords: college students, laboral precariousness, professional development.

\section{Introducción}

Uno de los efectos de las políticas neoliberales en el contexto de la globalización, ha sido la modificación del mercado laboral en detrimento de las condiciones laborales y consecuentemente del nivel de vida de los trabajadores. Según Beck (1998), teórico de la globalización, el capitalismo destruye el trabajo, el desempleo ya no es algo marginal en la vida económica de los países porque afecta potencialmente a todos y lo que se ha presentado como remedio es la llamada flexibilización del mercado laboral que en la práctica conduce a trabajos de tiempo parcial y precarias relaciones contractuales. El mismo Beck (2007), asegura que la explotación y la decadencia del trabajo se ha vuelto una precariedad masiva, evitando el crecimiento y desarrollo no sólo de los países menos desarrollados, sino forzando a los jóvenes a soportar condiciones de precariedad laboral.

En el caso de América Latina, los cambios en el mercado laboral están delimitados por la globalización y un modelo de acumulación de capital que se caracteriza por la desregulación y la flexibilidad laboral, mismas que conducen al deterioro de las condiciones laborales, aumento del trabajo por cuenta propia, transformación de los modelos de regulación laboral, entre otros cambios, (Mancini, 2014). En este contexto, los jóvenes se enfrentan a trayectorias laborales inestables, inseguras, con bajos ingresos o desempleo.

México es un país con altos niveles de pobreza y desigualdad, 57.7 millones de personas no cuenta con

1. En el desarrollo de este trabajo se contó con la participación de colaboradores del Cuerpo Académico CA UDG 525: Juan Alberto Gran Castro, Ulises Osbaldo de la Cruz Guzmán, Larisa Flores Valenzuela y Geomara Chávez.

2. Profesora e Investigadora titular C adscrita al Departamento de Ciencias Sociales y Jurídicas. CUCEA. Universidad de Guadalajara. Doctora en ciencias de la S. en el trabajo, maestra en ciencias de la educación y licenciada en psicología, por la Universidad de Guadalajara. Miembro del Sistema Nacional de Investigadores Nivel I en el Área de Ciencias Sociales, bsilva@ cucea.udg.mx

3. Profesora e Investigadora titular C adscrita al Departamento de Ciencias Sociales y Jurídicas. CUCEA. Universidad de Guadalajara. Doctora en educación superior por la Universidad de Guadalajara, Maestra en Ciencias Sociales-FLACSO, y Licenciada en Sociología por la Universidad de El Salvador 
los ingresos suficientes para satisfacer la canasta básica, por tanto, no es difícil inferir la situación de los jóvenes, especialmente cuando únicamente el $17.3 \%$ de los ubicados en el rango de edad de 14 a 29 años, forman parte de un hogar sin carencia económica o social (Mora y De Oliveira, 2014).

No es de extrañar entonces que los jóvenes inicien su acercamiento a la vida laboral al mismo tiempo que se dedican a su formación profesional en el nivel superior; este proceso involucra diversos elementos, que para este estudio serán analizados a partir de variables sociodemográficas, académicas, institucionales y las relativas a las características particulares del trabajo que desempeñan los estudiantes.

\section{Metodología}

El estudio tuvo lugar en el Centro Universitario de Ciencias Económico Administrativas de la Universidad de Guadalajara. Esta instituición ocupa el tercer lugar en matrícula total a nivel nacional (UNAM, 2015) y primer lugar estatal con 255,944 estudiantes en toda la Red Universitaria $^{4}$ (Bravo, Navarro, Peña y Moreno, 2015). Entre los centros universitarios de la Red, el de mayor matrícula es el de Ciencias de la Salud $(17,906)$, seguido del CUCEA (17,768), y después el de Ciencias Exactas e Ingenierías (13,840) (Bravo, et al., 2015). En el perido de la aplicación de la encuesta (2015) el CUCEA contó con una población de 17,768 estudiantes en el nivel de licenciatura. La investigación se realizó mediante un enfoque cuantitativo de alcance descriptivo e inferencial, a través del uso de medidas de tendencia central y regresiones lineales simples y ampliadas; con un corte transversal, utilizando la encuesta como herramienta de obtención de información.

El proceso de investigación contempló un periodo de aplicación de cuestionarios durante el segundo semestre del 2015. Debido a la magnitud de la población de estudiantes del CUCEA se optó por utilizar los medios electrónicos con el propósito de facilitar la aplicación del cuestionario, para ello se establecieron los criterios de inclusión tomando como base los porcentajes de créditos concluidos hasta el momento ${ }^{5}$, la Secretaría Académica proporcionó un directorio estudiantil para obtener los correos electrónicos de los estudiantes a fin de invitarlos a participar en el estudio. A partir de los criterios de inclusión se obtuvo el dato de 3,157 alumnos que conformaron la población objeto de estudio; con este número la muestra representativa con un nivel de confianza del $95 \%$, se integró con 346 estudiantes.

La encuesta se conformó por un cuestionario que en un primer momento incluyó los aspectos sociodemográficos del estudiante, enseguida los aspectos académicos y posteriormente los laborales. Los apartados de aspectos sociodemográficos y académicos son de elaboración propia y el de aspectos laborales, se tomó de la Encuesta Nacional de Ocupación y Empleo (ENOE) elaborada por el Instituto Nacional de Estadística y Geografía (INEGI) en su versión de "cuestionario básico".

El instrumento se administró de manera virtual mediante la plataforma google forms a partir de dos estrategias: envío por correo electrónico y aplicación directa en aulas de clase. Con base en lo anterior, se utilizó una pregunta filtro en el cuestionario para determinar si el estudiante cubría la característica de contar con un trabajo remunerado en los últimos seis meses. En caso de cumplir con dicho criterio, los estudiantes continuaban con el llenado del cuestionario, en caso contrario, el participante concluía su aportación. Se obtuvo un total de 145 estudiantes laboralmente activos.

Con la información obtenida se construyó una base de datos en Excel, cuyas estimaciones y cálculos se realizaron en SPSS (Statistical Package for the Social Sciences). Los datos académicos de los estudiantes, como promedio, porcentaje de créditos, número de materias cursadas por ciclo escolar, entre otras, se obtuvieron a través de la consulta del Sistema Integral de Información y Administración Universitaria (SIIAU), a partir del código de los estudiantes, para lo que se contó con el consentimiento de los mismos.

Se realizó el análisis descriptivo de los datos con el uso de las medidas de tendencia central. Posteriormente se codificó la base de datos: a la variable sexo se le otorgó el valor de cero para hombre y uno a mujer; la antigüedad en el trabajo se integró en cuatro rangos ${ }^{6}$ por lo que se decidió establecer la media de estos para el cálculo de

4. La Red Universitaria de la Universidad de Guadalajara se compone por seis centros universitarios (CU) temáticos ubicados en la zona metropolitana de Guadalajara y por nueve centros regionales que se encuentran en municipios de Jalisco. Los centros temáticos se integran por el CU de Ciencias de la Salud, Ciencias Sociales y Humanidades, Ciencias Biológicas y Agropecuarias, Ciencias Económica-Administrativas y Ciencias Exactas e Ingenierías y de Arte, Arquitectura y Diseño. Los centros regionales por el CU-Altos en Tepatitlán de Morelos, CU-Ciénega en Ocotlán, CU-Costa en Puerto Vallarta, CU-Valles en Ameca, CU-Lagos en Lagos de Moreno, CU-Ciénega en Ocotlán; dos CU-Sur en Autlán de Navarro y Ciudad Guzmán, CU-Norte en Colotlán y CU-Tonalá.

5. El criterio de inclusión considerado tomó el avance académico de los estudiantes a través de su porcentaje de créditos cursados, es decir, materias: de 30-39\% como inicio de la carrera, $60-69 \%$ como el periodo medio y $90-99 \%$ como cierre de su licenciatura.

6. De 6 meses a 1 año, de 1 a 2 años, de 3 a 4 años, de 5 a 6 años, de 7 a 8 años, de 9 a 10 años, mayor a 10 años y otros. 
las regresiones; se realizó el mismo procedimiento para las horas semanales dedicadas a su trabajo ${ }^{7}$ y el ingreso total mensual ${ }^{8}$; mientras que la edad se mantuvo con las respuestas originales.

Se utilizó un modelo de regresión lineal simple para conocer la relación existente entre el ingreso como variable dependiente, ya que es mencionada por Navarrete (2012), Sánchez (2014), entre otros, como un factor clave de medición de la precariedad, y como variables independientes las horas laboradas, edad y antigüedad, aplicando el análisis de forma individual; además de aplicar una regresión lineal ampliada para analizar la relación entre ingreso, hora y edad en conjunto.

\section{Trabajo y precariedad laboral}

La precariedad laboral en México es una situación generalizada para la mayoría de la juventud (Navarrete, 2012), ésta se entiende como una forma temporal de trabajo, ya que en el futuro pretende dirigirse hacia mejores situaciones laborales, como apuntan las denominadas trayectorias flexibles, aunque también puede transformarse en una condición permanente de relación con el mercado de trabajo, que generalmente es lo que lo sucede en el camino de muchos jóvenes (Santamaría, 2012).

Al respecto, Santamaría (2012) elaboró una clasificación de trayectorias juveniles divididas en: flexibles, precarias y precarizadas; las primeras hacen énfasis en una incertidumbre laboral en la que puede existir cierta continuidad en el trabajo, a pesar de los contratos temporales, una percepción monetaria baja pero con una estabilidad respecto al sector en el que se desenvuelven los jóvenes; la segunda trayectoria refleja la inestabilidad laboral de manera continua, a través de cambios de campo laboral, cambios de profesión e incluso el autoempleo; por último, las trayectorias precarizadas son definidas como aquellas en las que la inestabilidad laboral se vuelve una constante, no existiendo oportunidad alguna de salir del estancamiento y nulidad de todas las condiciones laborales. En este contexto, para el caso de México, García (2013) afirma que la precariedad en el trabajo asalariado no ha ido en detrimento, ha permanecido e incluso ha incrementado significativamente desde el primer decenio del siglo XXI. El autor mide la precariedad a través de indicadores como la ausencia de prestaciones sociales o de contratos permanentes, lo cual contribuye significativamente al aumento de la inseguridad laboral, promoviendo la falta de protección social y reducción salarial, exponiendo a un amplio sector de trabajadores a una situación de vulnerabilidad o incluso de exclusión laboral.

La precariedad implica vulnerabilidad social de amplios sectores de la población y el debilitamiento del trabajo como uno de los mecanismos básicos de integración y movilidad social (Rubio, 2010). El cuadro se torna dramático al enfocarse en la población juvenil, donde un entorno de escasez de empleo y desregulación laboral propician que la fuerza de trabajo más joven se enfrente a condiciones laborales precarias, Navarro (2001), citado por De Oliveira (2006).

Sin embargo, a pesar de la precariedad que se presenta en las condiciones del empleo juvenil, para Beduwe y Giret (2004), así como para Guzmán (2004), los estudiantes universitarios encuentran motivos para trabajar. Según Planas y Enciso (2014), existe una esfera de estudiantes que llevan a cabo su primer acercamiento hacia el ambiente laboral, partiendo de las necesidades económicas, el sentimiento de autonomía y autosuficiencia que es generado al recibir una remuneración económica, la adquisición de experiencia que permita el enriquecimiento de su currículum, o en algunos casos, combinar la actividad académica con el trabajo a fin de atender las exigencias de los empleadores para la obtención del grado en su desempeño profesional, así como para crear una vinculación de sus estudios con la vida profesional.

Estos autores sostienen que el segmento opuesto son aquellos estudiantes que encuentran motivos para no realizar una temprana vinculación con el sector de la profesionalización, en donde consideran que el tiempo que tienen no les es suficiente para realizar ambas actividades, consideran que las demandas institucionales en relación al tiempo de aprovechamiento académico, truncan las oportunidades de obtener un trabajo, y en algunos casos, consideran que la carrera les proporciona la suficiente experiencia, por lo que no es indispensable el acercamiento al ambiente laboral.

Carrillo y Ríos (2014) afirman que es importante tomar en consideración que no sólo influye la perspectiva individual sino también la familiar, enfatizan las necesidades de aspecto económico como medida predominante para decidir si los estudiantes universitarios trabajan o no, y en otras instancias también se encuentra la motivación por la experiencia profesional, obtener aprendizaje y, en algunos casos, lograr la independencia económica y familiar; en este sentido Aramburu y Fernández (1999) enfatizan que

7. Menos de 15 horas, de 15 a 34 horas, de 35 a 48 horas, más de 48 horas.

8. Menos de 2,103, entre 2,103 y 4,206, entre 6,309 y 8,412, entre 10,515 y 12,618 , entre 14,721 y 16,824 , entre 18,927 y 21,030 y más de 21.030 pesos mexicanos. 
la aspiración social a la educación universitaria es una motivación que no sólo surge de manera intrínseca, sino que es fomentada fuertemente a través de los vínculos familiares.

\subsection{Precariedad laboral de los jóvenes universitarios} mexicanos

De acuerdo con la Encuesta Nacional de Ocupación y Empleo (ENOE) para diciembre de 2015, en México, $59.5 \%$ de la población de 15 años y más es económicamente activa, frente a una tasa de desocupación nacional de $4.4 \%$ (INEGI, 2016). En este sentido, el $68.8 \%$ de la Población Económicamente Activa (PEA) del país opera como trabajador subordinado y remunerado en plaza o puesto fijo, el 21.8\% trabaja de manera independiente o por su cuenta sin contratar empleados, y el $4.4 \%$ labora en negocios o parcelas familiares y son empleadores o patrones (INEGI, 2016).

En el año 2006, De Oliveira (2006) destacaba que México había reducido significativamente su nivel de desempleo además de enfrentar un deterioro de las condiciones laborales así como de los ingresos percibidos, por lo que esto implicaba dificultades para la creación de empleos de calidad que absorban la cantidad de la fuerza laboral disponible. Pasan los años y la disminución de las tasas de desempleo no ha sido suficiente para mejorar las condiciones de precarización existentes (García, 2013); por ello es importante tener presente que las condiciones de los asalariados no son a favor de una seguridad y estabilidad laboral (Mora y De Oliveira, 2012).

De los datos de la PEA correspondiente al 2016, 95.4\% hace énfasis en la disponibilidad de tiempo para trabajar más horas, de acuerdo con su necesidad de percibir mayores ingresos para subsistir. A este sector de personas se les denomina subocupados (INEGI, 2016). De acuerdo con Beck (1998), el fenómeno de la subocupación es un medio de subsistencia, donde las condiciones laborales precarias no brindan ninguna estabilidad laboral.

Autores como Rodríguez e Ibarguren (2009), así como Rubio (2010) argumentan que la situación arriba mencionada, propicia que la población en edad de trabajar se desempeñe sin la calificación adecuada, aceptando empleos informales, sin un contrato laboral y de carácter temporal con carencia de prestaciones sociales. Tal es el caso de los jóvenes, quienes, según Sánchez (2014) y Santamaría (2012), se encuentran con una tasa de desempleo mayor en comparación con los adultos, por esta razón, cuando llegan a encontrar un trabajo remunerado, sus condiciones son precarias, debido a la falta de protección social, su temporalidad e inestabilidad. Paradójicamente los jóvenes que estudian suelen atravesar largas fases de educación y en algunos casos prefieren la continuación de los estudios debido a la falta de oportunidades laborales o se enfrentan a un mercado de trabajo que les exige experiencia laboral aun cuando el mercado no les permite acceder a un primer empleo, y en caso de lograrlo, las condiciones son de precariedad.

En los jóvenes universitarios, Navarrete (2012), encontró que aún cuando tienen la posiblidad de tener una educación formal a nivel superior tienen bajos salarios, contratos inexistentes o temporales, así como la falta de prestaciones en el trabajo, que como puede observarse, son características propias de la precariedad laboral. Entre sus hallazgos, encontró que los jóvenes que cuentan con una educación menos escolarizada, toman empleos en condiciones de mayor precariedad, mientras que aquellos que han tenido una escolarización más alta, como los universitarios, cuentan con una situación menos deteriorada, por lo que asegura que la formación universitaria pareciera no ser suficiente para atender las demandas del mercado laboral respecto al capital humano. Ante este panorama es importante señalar que la educación y el empleo guardan un vínculo esencial para el desarrollo de toda persona.

Según Navarrete (2012), comúnmente, los altos logros educativos son relacionados a compensaciones de buen empleo), pero esos logros dificilmente se observan mientras se realizan los estudios, mientras tanto, el empleo adquiere una doble noción, la de satisfacer las necesidades económicas del estudiante y, a su vez, complementar su formación académica. Al respecto, cabe mencionar la dificultad que presenta para los estudiantes conciliar ambas actividades, como lo demuestra el estudio de Silva, Rodríguez, Vicente y Leyva (2012) acerca de los factores que inciden en la reprobación de los estudiantes de licenciatura del CUCEA. El $72.5 \%$ de los estudiantes reprobados ( $10 \%$ de la población total), atribuye como causal de su situación al trabajo y la imposibilidad de abandonarlo.

\section{Discusión de resultados}

\subsection{Perfil sociodemográfico de los estudiantes}

Se obtuvo la participación de un total de 346, estudiantes del CUCEA, de los cuales, 145 (41.91\%) han tenido un trabajo remunerado en los últimos seis meses desde el periodo de aplicación de la encuesta. El resto, 201 (56.65\%) indicó que no contaba con trabajo (Cuadro $\mathrm{N}^{\mathrm{o}} 1$ ).

A continuación, se describe el perfil sociodemográfico de los encuestados laboralmente activos. 
Cuadro $N^{\circ}$ 1. CUCEA: perfil sociodemográfico de estudiantes encuestados

\begin{tabular}{|c|c|c|c|c|c|}
\hline Variables & Frecuencia & Porcentaje & Media & Moda & Rango \\
\hline $\begin{array}{c}\text { Edad/años } \\
\text { cumplidos }\end{array}$ & & & 23.76 & 22 & $19-39$ \\
\hline $\begin{array}{c}\text { Sexo } \\
\text { Hombre }\end{array}$ & 66 & 45.5 & & & \\
\hline Mujer & 76 & 54.5 & & & \\
\hline Total & $\mathbf{1 4 5}$ & $\mathbf{1 0 0}$ & & & \\
\hline Est. cívil & & & & & \\
\hline Casado & 6 & 4.1 & & & \\
\hline Divorciado & 1 & 0.7 & & & \\
\hline Soltero & 129 & 89.0 & & & \\
\hline Unión libre & 9 & 6.2 & & & \\
\hline Total & $\mathbf{1 4 5}$ & $\mathbf{1 0 0 . 0}$ & & & \\
\hline
\end{tabular}

Fuente: Elaboración propia en base a datos de encuesta

4.2 Caracterización de la vida académica de los estudiantes

Los resultados de la encuesta señalan que los estudiantes del CUCEA se enfrentan a un reto ante la distribución del tiempo, por cuestiones como los horarios en que se imparten las materias y traslados entre la universidad y el trabajo. $47.59 \%$ utilizan entre 1 y 2 horas para trasladarse diariamente, y $33.10 \%$ entre 3 y 4 horas. Sumado a esto, en su mayoría ( $81.38 \%$ ), dedican menos de ocho horas semanales al estudio y a las tareas relacionadas a sus materias. El promedio general de calificaciones de la muestra es de 85.56 , con una moda de 77.08 sobre 100 (Cuadro $\mathrm{N}^{\circ}$ 2).

Cuadro $\mathrm{N}^{\circ}$ 2. CUCEA: horas a la semana que los estudiantes entrevistados dedican a estudiar y hacer tareas

\begin{tabular}{|c|c|c|}
\hline Nímero de horas & Frecuencia & Porcentaje \\
\hline 1 & 5 & 3.45 \\
\hline 2 & 13 & 8.97 \\
\hline 3 & 22 & 15.17 \\
\hline 4 & 18 & 12.41 \\
\hline 5 & 19 & 13.10 \\
\hline 6 & 18 & 12.41 \\
\hline 7 & 9 & 6.21 \\
\hline 8 & 14 & 9.66 \\
\hline Más de 8 & 27 & 18.62 \\
\hline Total & $\mathbf{1 4 5}$ & $\mathbf{1 0 0}$ \\
\hline
\end{tabular}

Fuente: Elaboración propia en base a datos de encuesta

En promedio, los encuestados toman seis materias semanalmente, cada una consta de cuatro horas de clase presencial por semana. El horario en que asisten a la universidad es en su mayoría vespertino $(38.62 \%)$, aunque resaltan numerosos casos de horario matutino $(31.03 \%)$ y mixto $(30.34 \%)$, que son quienes asisten a clase durante mañana y tarde. Sumado a esto, los estudiantes en periodos más avanzados, deben cumplir con un servicio social y prácticas profesionales para concluir la formación profesional: $19.31 \%$ y $6.89 \%$ de los alumnos realizan prácticas y servicio, respectivamente, además de atender sus materias y el trabajo.

Asistir a clases, estudiar y realizar tareas, trabajar, trasladarse del trabajo a la universidad o viceversa, cumplir con el servicio social y prácticas profesionales, se muestran como determinantes de la distribución del tiempo para estos jóvenes. Frente a esta carga de responsabilidades, el descanso y recreación, esenciales para el desempeño académico, se ve disminuido; resalta que el $77 \%$ dedica 3 o menos horas de ocio al día y $54.5 \%$ duerme entre 3 y 5 horas.

4.3 Caracterización de la situación laboral de los estudiantes.

Respecto a la situación laboral de los estudiantes del CUCEA, los resultados muestran que existen condiciones de precariedad laboral; ante esto, expresan que el objetivo de tener un trabajo es principalmente pagar sus estudios, cubrir gastos propios de sostenimiento y contribuir a gastos familiares (Gráficos $\mathrm{N}^{\circ} 1$ y No 2 ). Realizan trabajos como auxiliar en actividades administrativas (44.83), centradas al comercio y ventas $(20.69 \%)$ y profesionistas técnicos $(13.10 \%)$. Además, son empleados por empresas privadas $(66.90 \%)$, seguido del negocio familiar $(17.93 \%)$ y por la institución pública $(8.97 \%)$, el resto cuenta con negocio propio. Finalmente, sus trabajos se ubican en el sector de servicios $(41.38 \%)$ y comercio $(26.21 \%)$.

\section{Gráfico $N^{0} 1$. CUCEA: motivo por el que trabajan los estudiantes entrevistados}
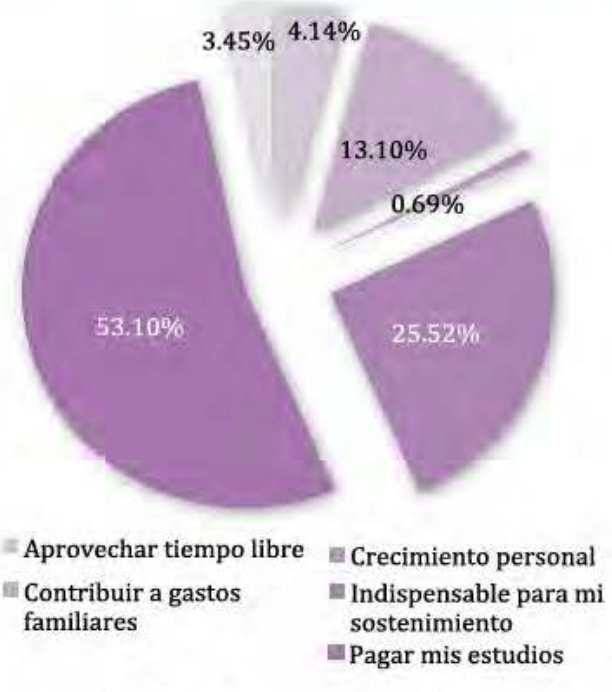

Fuente: Elaboración propia en base a datos de encuesta 
Gráfico $N^{\circ}$ 2. CUCEA: motivo por el que trabajan, adicional al pago de los estudios

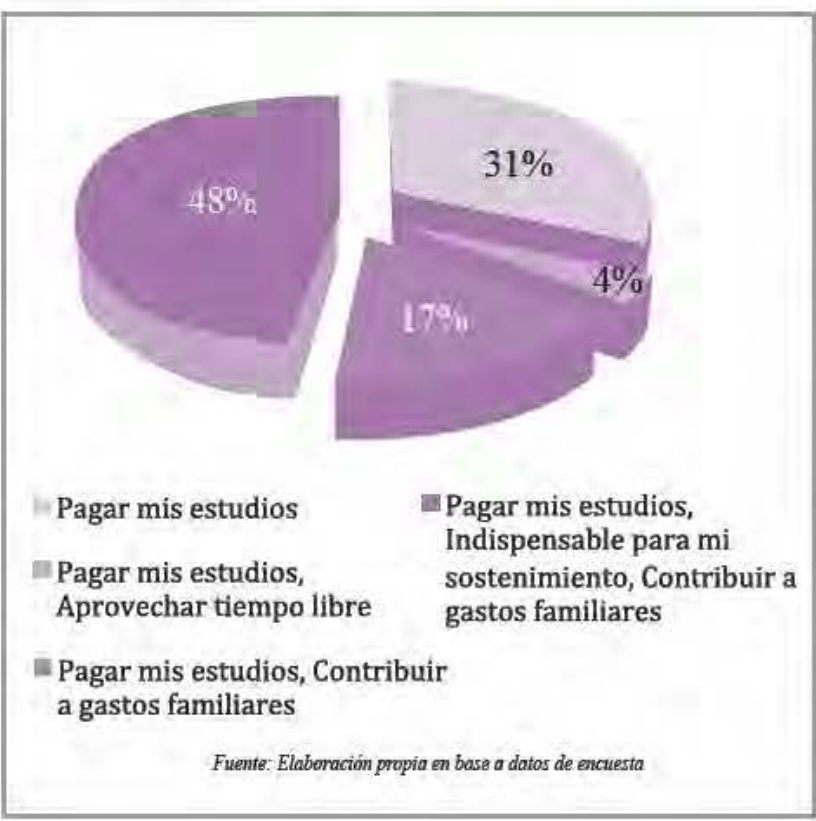

Según la percepción de los estudiantes, $59.31 \%$ manifiestan que su trabajo sí está relacionado con su carrera y el resto comenta lo contrario. Si se toma en cuenta la condición de estudiante, es considerable el tiempo que llevan desempeñando actividades remuneradass, pues, en su mayoría afirman tener una antigüedad laboral entre 1 y 2 años (53.10\%), seguido de 3 a 4 años (17.93\%) y de 6 meses a 1 año $(15.17 \%)$, entre otros periodos para el resto de los encuestados.

En cuanto a las condiciones laborales de los estudiantes, el Cuadro $\mathrm{N}^{\circ} 3$ muestra algunas variables de importancia. La mayor parte de ellos trabaja con una jornada laboral de medio tiempo, aunque existen casos de tiempo completo. La formalidad de los trabajos es baja, ya que la mayoría son los denominados "de confianza", es decir, que son empleados no sindicalizados con contratos temporales o "indefinidos" en el sentido temporal de permanencia, o incluso no tienen contrato, las prestaciones son pocas e incluso inexistentes; sólo $1.4 \%$ de los encuestados son acreedores de las tres prestación básicas establecidas en la Ley Federal del Trabajo: vivienda, cesantía y vejez, y seguridad social (Cámara de Diputados, 2015); 24.1\% tienen únicamente prestación de vivienda y $3.4 \%$ de cesantía y vejez, mientras que $17.9 \%$ tiene ambas. Por un lado lado, $4.1 \%$ cuenta con gastos médicos privados y sólo $14.5 \%$ tiene este seguro y las prestaciones básicas de ley.
De quienes sí cuentan con las prestaciones de ley, $68.2 \%$ no tienen seguridad médica, por lo que ellos deben solventar sus gastos personales de salud. Cabe señalar, que $35.9 \%$ no tienen ninguna prestación. Por otro lado, el $12.41 \%$ tiene prestaciones superiores a la ley'.

El ingreso mensual de los estudiantes se encuentra principalmente en un rango que va entre 2,103 y 4,206 pesos mexicanos, lo cual equivale entre 118.41 y 247.41 dólares aproximadamente ${ }^{10}$ (Cuadro $\mathrm{N}^{0} 7$ ). Cabe mencionar que el salario mínimo para 2016 en Jalisco, alcanza la suma de 73.04 pesos diarios (CONASAM, 2016). Agregado a la dificultad de la distribución del tiempo dentro del aspecto académico, en la parte laboral, los estudiantes del CUCEA encuentran como reto la administración de gastos personales.

\section{Cundro $\mathrm{N}^{*}$ 3. CUCEA: características de la condición laboral} estudiantil entre estudiantes entrevistados

\begin{tabular}{|l|l|l|}
\hline Aspectos seleccionados & Frecuencia & Poircentaje \\
\hline Jornada laboral & & \\
\hline Tiempo completo & 47 & 32.41 \\
\hline Medio tiempo & 71 & 48.96 \\
\hline Fines de semana & 11 & 7.59 \\
\hline Eventual & 8 & 5.52 \\
\hline Otro & 8 & 5.52 \\
\hline Total & 145 & 100 \\
\hline Lagar de trabajo & & \\
\hline Sitio cerrado & 137 & 94.48 \\
\hline Sitio abierto & 8 & 5.52 \\
\hline Total & 145 & 100 \\
\hline Tipo de empleado & & \\
\hline De confianza & 96 & 66.21 \\
\hline Sindicalizado & 14 & 9.66 \\
\hline No sé & 34 & 23.45 \\
\hline Total & 144 & 99.32 \\
\hline Tipo de contrato & & \\
\hline De base, planta o tiempo & 61 & 42.07 \\
\hline indefinido & 45 & 31.03 \\
\hline Sin contrato escrito & 33 & 22.7 \\
\hline Temporal & 6 & 4.2 \\
\hline Otro & 145 & 100 \\
\hline Total & & \\
\hline
\end{tabular}

Fuente: Elaboración propia en base a datos de encuesta

Para tratar de analizar la relación existente entre el ingreso, como variable dependiente y factor predominante en la precariedad laboral y otras variables como la edad, la antigüedad y las horas laboradas semanalmente, se trabajaron modelos de regresión, específicamente tres modelos de regresión: lineal simple y uno compuesto.

9. Por prestaciones prestaciones superiores a la ley se toman bonos, seguro de vida y préstamos personales y caja de ahorro. El cálculo porcentual de las prestaciones presentó un dato perdido. 10. Tipo de cambio tomado de Banco de México: http//www.banxico.org mx/dyn/portal-mercado-cambiario/index html, al 3 de abril de 2016. 
- Modelo 1: edad e ingreso

Se hizo un modelo de regresión simple para buscar la relación existente entre edad e ingreso, quedando expresado de la siguiente manera:

$$
\text { Fing }=C+\beta_{1}+\beta_{2}+\ldots .+\beta_{145}+\varepsilon
$$

- Ing: ingreso percibido por los alumnos

- C: la Constante del modelo

- $\quad \beta 1>0$

- $\quad \varepsilon$ : es el error estándar de la regresión.

De lo anterior se obtuvo que por cada año más de edad que se tenga el ingreso tenderá a incrementarse en $\$ 1,878.75$ mensualmente. El $\mathrm{R}$ cuadrado muestra que por que cada año de edad el salario se modificara en un $10.5 \%$, con una significancia estadística a niveles del $99 \%$. Además de que el estadístico $t$ muestra que hay una correlación positiva entre las variables (Cuadro $\mathrm{N}^{\circ} 4$ ).

\begin{tabular}{|c|c|c|c|}
\hline Modelo & B & Error estándar & $\mathrm{T}$ \\
\hline Constante & $1,878.75$ & $1,670.633$ & 1.125 \\
\hline Edad & 286.116 & 69.767 & 4.101 \\
\hline R cuadrado & 0.105 & Sig. & 0.000 \\
\hline
\end{tabular}

Fuente: Elaboración propia en base a datos de encuesta

- Modelo 2: hora laboral e ingreso

Se realizó por separado un modelo de regresión simple para buscar la relación existente entre hora e ingreso:

$$
\text { Fing }=C+\beta_{1}+\beta_{2}+\ldots .+\beta_{145}+\varepsilon
$$

- Ing: ingreso percibido por los alumnos

- $\quad$ C: la Constante del modelo

- $\quad \beta 1>0$

- $\quad \varepsilon:$ es el error estándar de la regresión.

Seencontróqueporcadahora más que se trabajaa la semana el ingreso tendrá un incremento de $\$ 755.326$ mensualmente. El $R$ cuadrado muestra que por que cada hora de trabajo el salario se modificara en un $19.3 \%$, con una significancia estadística a niveles del $99 \%$ (Cuadro $\mathrm{N}^{\circ} 5$ ).

Además de que el estadístico t muestra que hay una correlación positiva entre las variables.
Cuadro $\mathrm{N}^{0} 5$. Estadísticos del modelo de regresión hora laboral e ingreso

\begin{tabular}{|l|c|c|c|}
\hline \multicolumn{1}{|c|}{ Modelo } & B & Error estándar & T \\
\hline Constante & $\mathbf{7 5 5 . 3 2 6}$ & $\mathbf{7 5 2 . 3 1 6}$ & 1.004 \\
\hline Edad & 129.931 & 22.233 & 5.844 \\
\hline R cuadrado & 0.193 & Sig. & 0.000 \\
\hline
\end{tabular}

Fuente: Elaboración propia en base a datos de encuesta

- Modelo 3: antigüedad laboral e ingreso

A través de la regresión simple se generó un modelo para analizar la relación existente entre antigüedad e ingreso:

$$
F \text { ing }=C+\beta_{1}+\beta_{2}+\ldots .+\beta_{145}+\varepsilon
$$

- Ing: ingreso percibido por los alumnos

- C: la Constante del modelo

- $\quad \beta 1>0$

- $\quad \varepsilon$ : es el error estándar de la regresión.

Se obtuvo que por cada año más de antigüedad, el ingreso tenderá a incrementarse en \$ 4,202.79 mensuales. El $\mathrm{R}$ cuadrado muestra que por cada año de antigüedad que se tenga de más, el salario se modificara en un $2.5 \%$, con una significancia estadística a niveles del $90 \%$ (Cuadro $\mathrm{N}^{\circ} 6$ ).

Además de que el estadístico t muestra que hay una correlación positiva entre las variables

Cuadro $N^{\circ} 6$. Estadísticos del modelo de regresión antiguedad $\mathrm{e}$ ingreso

\begin{tabular}{|l|c|c|c|}
\hline \multicolumn{1}{|c|}{ Modelo } & B & Error estándar & T \\
\hline Constante & 4202.793 & 457.448 & 9.187 \\
\hline Antiguedad & 275.629 & 145.228 & 1.898 \\
\hline R cuadrado & 0.025 & Sig. & 0.060 \\
\hline
\end{tabular}

Fuente: Elaboración propia en base a datos de encuesta

El incremento en el ingreso por cada año más de antigüedad es sustancial por lo que es posible señalar que la precariedad en los trabajos de los jóvenes estudiantes es muy poca si se toma al ingreso como referencia; sin embargo en el siguiente cuadro se constata el por qué de este resultado; ya que la mayoría de los entrevistados tienen una antigüedad mayor a 1 año y se encuentran distribuidos a partir de 1 a 2 salarios mínimos hasta el mayor a 10 salarios. De manera más específica, aquellos que tienen una antigüedad mayor a 1 año y tienen un ingreso que va de 3 a más de 10 salarios mínimos, hallamos que representan el $32 \%$ de los estudiantes (Cuadro $\mathrm{N}^{\circ} 7$ ). 
Cuadro $\mathrm{N}^{\circ}$ 7. CUCEA: cruce de frecuencias entre antiguedad e ingreso de los estudiantes entrevistados

\begin{tabular}{|c|c|c|c|c|c|c|c|c|}
\hline \multirow[b]{2}{*}{ Antiguedad } & \multicolumn{6}{|c|}{ Ingreso mensual personal } & \multirow[b]{2}{*}{$\begin{array}{l}\text { Total } \\
\text { de casos }\end{array}$} & \multirow[b]{2}{*}{ Porcentaje } \\
\hline & $\begin{array}{c}\text { Menor a } \\
\text { un SM }\end{array}$ & 1 a $2 \mathrm{SM}$ & 3 a 4 SM & 5 a $6 \mathrm{SM}$ & 7 a $8 \mathrm{SM}$ & $\begin{array}{l}\text { Mayor a } \\
10 \mathrm{SM}\end{array}$ & & \\
\hline $0.6-1$ & 6 & 11 & 3 & 1 & 0 & 1 & 22 & $15 \%$ \\
\hline $1-2$ & 8 & 47 & 17 & 4 & 1 & 0 & 77 & 53 \\
\hline $3-4$ & 3 & 6 & 14 & 3 & 0 & 0 & 26 & 18 \\
\hline $5-6$ & 2 & 6 & 2 & 1 & 1 & 0 & 12 & 8 \\
\hline $7-8$ & 1 & 2 & 0 & 2 & 0 & 0 & 5 & 3 \\
\hline $9-10$ & 0 & 1 & 0 & 0 & 0 & 0 & 1 & 1 \\
\hline Mayor a 10 & 0 & 0 & 2 & 0 & 0 & 0 & 2 & 1 \\
\hline \multirow[t]{2}{*}{ Total } & 20 & 73 & 38 & 11 & 2 & 1 & & \\
\hline & $14 \%$ & $50 \%$ & $26 \%$ & $8 \%$ & $1 \%$ & $1 \%$ & 145 & 100 \\
\hline
\end{tabular}

Fuente: Elaboración propia en base a datos de encuesta

\section{- Modelo 4: edad, horas laboradas e ingreso}

También se elaboró un modelo compuesto en donde se buscaba conocer la relación existente entre edad, horas laboradas y la antigüedad, con el ingreso como variable dependiente; no obstante la significancia de la antigüedad se vio mermada, por lo que no se consideró relevante y se eliminó del modelo ampliado, dejando como objetivo en esta regresión el analizar la relación existente entre el Ingreso y las variables en conjunto de edad y horas laboradas:

$$
\begin{gathered}
\text { Fing }=C+\beta_{1} \text { Edad }_{i}+\beta_{2} \text { Horas }_{i}+\varepsilon \\
i=1,2, \ldots \ldots, 145
\end{gathered}
$$

- Ing: ingreso percibido por los alumnos

- C: la Constante del modelo

- $\quad \beta 1$ Edadi $>0$

- $\quad \beta 2$ Horasi $>0$

- $\quad \varepsilon$ : es el error estándar de la regresión.

Este modelo arrojó que por cada año de edad más y cada hora adicional que se labore semanalmente el ingreso mostrará un incremento de $\$ 3,639.12$ mensualmente. El $\mathrm{R}$ cuadrado muestra que por que cada año de edad y por cada hora de trabajo adicional semanalmente el salario se modificara en un $24.5 \%$, con una significancia estadística arriba del $95 \%$ (Cuadro $\mathrm{N}^{\circ} 8$ ). Además de que el estadístico $t$ muestra que hay una correlación positiva entre las variables.
El estadístico F Fisher y Snedecor muestra que el modelo en su conjunto es estadísticamente significativo

\begin{tabular}{|c|c|c|c|}
\hline Modelo & B & Error estándar & $\mathrm{T}$ \\
\hline Constante & 3639.12 & 1577.461 & 2.307 \\
\hline Edad & 207.690 & 66.090 & 3.143 \\
\hline Horas laborales & 113.822 & 22.174 & 5.133 \\
\hline R cuadrado & 0.245 & $\mathrm{~F}$ & 23.074 \\
\hline
\end{tabular}
puesto que es del 23.074.

Fuente: Elaboración propia en base a datos de encuesta

\section{Conclusiones}

La situación laboral de los estudiantes refleja factores de precariedad puesto que más de la mitad de los encuestados expresaron contar con empleos temporales o sin contrato, por consecuencia, las prestaciones son pocas e incluso inexistentes; además de percibir bajo sueldo (entre 2, 103 y 4, 206 pesos mexicanos al mes), sin embargo se encontró que este porcentaje se distribuye en los rangos de antigüedad de 6 meses a 1 año y de 1 a 2 años, es decir, que se trata de estudiantes de reciente incorporación al trabajo. Esta situación es un reflejo de lo establecido en el artículo 39-A de la Ley Federal del Trabajo, que señala que los empleadores pueden tener a sus trabajadores en un periodo de capacitación inicial de tres meses más un periodo de prueba del mismo tiempo, en el que los sueldos suelen ser menores.

Los datos obtenidos a través de los modelos de regresión lineal muestran que a partir de la experiencia laboral, a 
mayor número de horas dedicadas al trabajo y a mayor edad, el ingreso de los estudiantes incrementará. Lo cual confirma que al tener una permanencia mayor en el lugar de trabajo y generar antigüedad, el salario crecerá proporcionalmente.

El desarrollo del estudio también determinó que el desempeño académico en los universitarios del CUCEA que trabajan, muestra un promedio general de calificaciones de 85.56 sobre 100 y moda de 77 sobre 100 . Uno de los mayores retos de los universitarios del CUCEA es mantener un promedio alto frente a la distribución del tiempo, numerosas responsabilidades académicas, jornadas laborales y traslados que realizan entre el lugar de estudio y trabajo. Estos elementos conducen a plantear nuevos cuestionamientos acerca del nivel de exigencia de los programas académicos y los criterios para aprobar los cursos, ya que los estudiantes expresaron dedicar muy pocas horas de estudio y realización de tareas durante la semana, además de que cursan 6 materias por semestre.

Así mismo, en razón de los escasos estudios que abordan la precariedad laboral desde la situación de los jóvenes universitarios, es evidente la necesidad de analizar con mayor profundidad este tema, sobre todo en instituciones educativas públicas y de gran presencia en cuanto a matrícula y oferta educativa, como es la Universidad de Guadalajara.

Finalmente, es importante señalar que frente a las demandas del mercado laboral en el contexto de la globalización y los procesos económicos neoliberales, la Universidad de Guadalajara, en el Centro de Ciencias Económico-Administrativas, requiere continuar innovando las estrategias de vinculación empresa-universidadgobierno, para que brinden mejores oportunidades a los jóvenes universitarios, a través de programas más eficientes de prácticas profesionales y bolsa de trabajo, lo cual representa los cimientos para mayores y mejores oportunidades laborales, en el entendido que es necesario profundizar en el contexto y las causas de esta situación.

\section{Bibliografía}

- Aramburu, L., Fernández J., (1999). Los jóvenes universitarios y el empleo. Boletín Ciudades para un Futuro más Sostenible. Madrid, España.

- Beck, U., (1998). La sociedad del riesgo. Hacia una nueva modernidad. Barcelona: Paidós.

- Beck, U., (2007). “Un nuevo mundo feliz. La precariedad en la era de la globalización”. Barcelona: Paidós.

- Béduwé, C., y Giret, J., (2004). Le travail en cours d'études a-t-il une valeur professionnelle?. Economie et Statistique. 378 (379): 55-83.
- Bravo, I.T., Navarro, M. A., Peña, J.A. y Moreno, C.I. (2015). Estadística institucional 2014-2015. Guadalajara: Universidad de Guadalajara.

- Cámara de Diputados (2015). Ley Federal del Trabajo. México, publicada en Diario Oficial de la Federación 1/04/1970. Última reforma al 12/06/2015.

- Carrillo, R., Ríos A., (2014). Oferta de trabajo de los estudiantes de la Universidad de Guadalajara y de México. Un análisis comparativo. Perfiles Educativos. 36 (144): 85-104.

- De Oliveira, O., (2006). Jóvenes y precariedad laboral en México. Papeles de población, 12 (49): 1405-1425.

- García, G., (2013). Precariedad laboral y desempleo en México. En Valdés, Luz María (2013) (coord.) "Hacia una nueva ley general de población”. México: UNAM (157- 177).

- Guzmán, C. (2004). Los estudiantes frente a su trabajo. Un análisis en torno a la construcción del sentido del Trabajo. Revista Mexicana de Investigación Educativa, 9 (22): 747-767.

- INEGI (2016). Indicadores de ocupación y empleo. Boletín de prensa número 22/16. 25 de enero de 2016. Consultado en: http://www.inegi.org.mx/saladeprensa/ boletines/2016/iooe/iooe2016_01.pdf

- Mancini, F (2014). El impacto de la incertidumbre laboral sobre el curso de vida durante la transición a la adultez. En ¿Ruptura o reproducción de las desventajas sociales heredadas?. Relatos de vida de jóvenes que han vivido situación de pobreza. En Mora, R y De Olivera (coord). Desafíos y Paradojas. Los jóvenes frenta a las desigualdades sociales. México. El Colegio de México.

- Mora, S., De Oliveira, O., (2012). "La situación del trabajo en México 2012, el trabajo en la crisis” De la Garza (Coord). Ed. Plaza y Valdés.

- Mora, S, y De Oliveira (2014). ¿Ruptura o reproducción de las desventajas sociales heredadas?. Relatos de vida de jóvenes que han vivido situación de pobreza. En Mora, R y De Olivera (coord). Desafíos y Paradojas. Los jóvenes frenta a las desigualdades sociales. México. El Colegio de México.

- Navarrete, E., (2012). Jóvenes universitarios mexicanos ante el trabajo. Revista Latinoamericana de Población, 6 (10): 119-140.

- Organización Internacional del Trabajo OIT (2005). El empleo de los jóvenes: vías para acceder a un trabajo decente. Suiza: OIT.

- Rodríguez, O. E., Ibarguren, L. S., (2009). Construcción de un índice de condiciones laborales por estados para 
México. Gestión y Política Pública, 58 (1): 149-178.

- Planas, C. J., Enciso, Á. M., (2014). Los estudiantes que trabajan: ¿Tiene valor profesional el trabajo durante los estudios? Universia, 12 (5): 23-45.

- Rubio, C., (2010). Precariedad Laboral en México. Una propuesta de medición integral. Revista Enfoques: Ciencia Política y Administración Pública. 8(3): 77-87.

- Sánchez, C. A., (2014). Los jóvenes frente al empleo y el desempleo: la necesaria construcción de soluciones multidimensionales y multifactoriales. Revista Latinoamericana de Derecho Social, 19: 133-162.

- Santamaría, L. E., (2012). Jóvenes y precariedad laboral: trayectorias laborales por los márgenes del empleo. Zerbituzuan, 52: 129-139.

- Silva-Gutiérrez, B. N., Rodríguez-Téllez, A. L., Vicente-Flores, R., Leyva-Ureña, H. (2012). Opinión de los estudiantes acerca de los motivos de reprobación en las licenciaturas del Centro Universitario de Ciencias Económico Administrativas (CUCEA). Revista Pequén. 2(1): 185-204. Universidad del Bio Bio.

- Universidad Nacional Autónoma de México [UNAM] (2015). Estudio comparativo de las universidades mexicanas. Explorador de Datos. Disponible en: http:// www.execum.unam.mx/ 